\title{
Potential Ways to Address Shortage Situations of ${ }^{99} \mathrm{Mo} /{ }^{99 m} \mathrm{Tc}$
}

\author{
Leah M. Filzen, Lacey R. Ellingson, Andrew M. Paulsen, and Joseph C. Hung \\ Division of Nuclear Medicine, Department of Radiology, Mayo Clinic, Rochester, Minnesota
}

\begin{abstract}
CE credit: For CE credit, you can access the test for this article, as well as additional JNMT CE tests, online at https://www.snmmilearningcenter.org. Complete the test online no later than March 2020. Your online test will be scored immediately. You may make 3 attempts to pass the test and must answer $80 \%$ of the questions correctly to receive $1.0 \mathrm{CEH}$ (Continuing Education Hour) credit. SNMMI members will have their CEH credit added to their VOICE transcript automatically; nonmembers will be able to print out a CE certificate upon successfully completing the test. The online test is free to SNMMI members; nonmembers must pay $\$ 15.00$ by credit card when logging onto the website to take the test.
\end{abstract}

\begin{abstract}
99mTc, the most common radioisotope used in nuclear medicine, is produced in a nuclear reactor from the decay of ${ }^{99} \mathrm{Mo}$. There are only a few aging nuclear reactors around the world that produce ${ }^{99} \mathrm{Mo}$, and one of the major contributors, the National Research Universal (Canada), ceased production on October 31, 2016. The National Research Universal produced approximately $40 \%$ of the world's ${ }^{99}$ Mo supply, so with its shut down, shortages of ${ }^{99} \mathrm{Mo} / 99 \mathrm{mTc}$ are expected. Methods: Nuclear pharmacies and nuclear medicine departments throughout the United States were contacted and asked to provide their strategies for coping with a shortage of ${ }^{99} \mathrm{Mo} /{ }^{99 \mathrm{~m} T c}$. Each of these strategies was evaluated on the basis of its effectiveness for conserving $99 \mathrm{mT} c$ while still meeting the needs of the patients. Results: From the responses, the following 6 categories of strategies, in order of importance, were compiled: contractual agreements with commercial nuclear pharmacies, alternative imaging protocols, changes in imaging schedules, software use, generator management, and reduction of ordered doses or elimination of backup doses. Conclusion: The supply chain of ${ }^{99} \mathrm{Mo} / 99 \mathrm{mTc}$ is quite fragile; therefore, being aware of the most appropriate coping strategies is crucial. It is essential to build a strong collaboration between the nuclear pharmacy and nuclear medicine department during a shortage situation. With both nuclear medicine departments and nuclear pharmacies implementing viable strategies, such as the ones proposed, the amount of ${ }^{99 \mathrm{~m} T c}$ available during a shortage situation can be maximized.
\end{abstract}

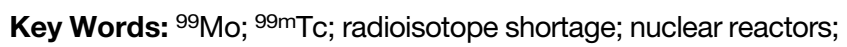
radioisotope productions

J Nucl Med Technol 2017; 45:1-5

DOI: 10.2967/jnmt.116.185454

$\mathbf{T}$ echnetium-99m is a principal radioisotope used for medical diagnostic imaging and accounts for approximately $80 \%$ of all nuclear medicine procedures $(1) .{ }^{99 \mathrm{~m}} \mathrm{Tc}$ is the daughter product of ${ }^{99} \mathrm{Mo}$, and it is typically supplied in the

Received Oct. 11, 2016; revision accepted Dec. 7, 2016.

For correspondence or reprints contact: Joseph C. Hung, Department of

Radiology, Mayo Clinic, 200 First St. SW, Rochester, MN 55905-0001.

E-mail: jhung@mayo.edu

Published online Feb. 2, 2017.

COPYRIGHT @ 2017 by the Society of Nuclear Medicine and Molecular Imaging. form of a ${ }^{99} \mathrm{Mo} /{ }^{99 m} \mathrm{Tc}$ generator to make diagnostic radiopharmaceuticals.

The global supply chain of ${ }^{99}$ Mo used for generator production has numerous complex problems, which makes the system fragile and unreliable. Limited suppliers, aging reactors, and transportation obstacles are all challenges faced with the supply chain (2). ${ }^{99} \mathrm{Mo}$ has been exclusively produced in 7 nuclear reactors, with an average age of these reactors being more than $40 \mathrm{y}$ old (2). These 7 nuclear reactors are the Belgian Reactor 2 (BR-2) in Belgium, High Flux Reactor in The Netherlands, LVR-15 REZ Reactor in the Czech Republic, Maria Research Reactor in Poland, National Research Universal (NRU) in Canada, Open Pool Australian Lightwater reactor in Australia, and South African Fundamental Atomic Research Installation in South Africa (2). In recent history, these reactors have encountered unscheduled maintenance and refurbishment periods impacting the supply of ${ }^{99} \mathrm{Mo}$.

The 7 nuclear reactors provide over $90 \%$ of the world's ${ }^{99}$ Mo (2). Global shortages of ${ }^{99 m} \mathrm{mc}$ emerged in 2009 due to 2 aging nuclear reactors (NRU in Canada and High Flux Reactor in The Netherlands) shutting down. Currently, the OSIRIS (France) has ceased production permanently. The BR-2 (Belgium) initiated a 12-mo refurbishment early last year with another projected 6 mo to complete the rebuild. The NRU ended its ${ }^{99}$ Mo production on October 31, 2016 (3). Any ${ }^{99}$ Mo production from the NRU after this date is subject to the discretion of the Canadian government (3). It has been proposed to keep the NRU in hot standby until March 31, 2018 (3). The Canadian government has not released the decisionmaking process for restarting ${ }^{99} \mathrm{Mo}$ production from the NRU after it ceased routine ${ }^{99}$ Mo production on October 31, 2016.

After being produced in nuclear reactors, ${ }^{99} \mathrm{Mo}$ is transferred to a processing facility to be chemically separated and purified. There are currently only $5{ }^{99}$ Mo-processing facilities in the world, which are Australian Nuclear Science and Technology Organization (ANSTO) in Australia, The Institute for Radio Elements (IRE) in Belgium, Mallinckrodt in The Netherlands, Nordion in Canada, and Nuclear Technology Products (NTP) Radioisotopes SOC Ltd. in South Africa (2). The finished ${ }^{99}$ Mo product material is then isolated and shipped to 1 of 8 generator-manufacturing facilities that supply 
${ }^{99} \mathrm{Mo}$ in the form of a ${ }^{99} \mathrm{Mo} /{ }^{99 m} \mathrm{Tc}$ generator to end users, such as nuclear pharmacies and hospitals (2). The 8 generatormanufacturing facilities are ANSTO (Australia), GE Healthcare (United Kingdom), IBA Molecular (France), Lantheus Medical Imaging (United States), Mallinckrodt (The Netherlands and United States), Monrol (Turkey), National Centre for Nuclear Research (NCBJ) Radioisotope Centre POLATOM (Poland), and NTP Radioisotopes SOC Ltd. in South Africa $(2,4)$.

In addition to the fragile reactor and ${ }^{99} \mathrm{Mo}$-processing facility infrastructures, the time frame to deliver purified ${ }^{99} \mathrm{Mo}$ to generator manufacturers is limited. Major transportation obstacles such as customs, government regulations, flight schedules, weather delays, pilot refusal, and natural disasters have the potential to result in significant product shipment delay (2).

It is difficult to predict when the next major ${ }^{99} \mathrm{Mo} /{ }^{99 m} \mathrm{Tc}$ shortage will take place. As such, it is important for each nuclear medicine facility to develop strategies to deal with periods of shortage. The purpose of this study was to identify potential approaches to manage shortage situations of ${ }^{99} \mathrm{Mo} /{ }^{99 m} \mathrm{Tc}$.

\section{MATERIALS AND METHODS}

Strategies for managing ${ }^{99} \mathrm{Mo} /{ }^{99 \mathrm{~m}} \mathrm{Tc}$ in shortage situations were gathered and pooled from a variety of sources. First, research was performed to gain information about the ${ }^{99}$ Mo supply chain, including information about the 7 nuclear reactors, the 5 processing facilities, the 8 generator-manufacturing facilities, and the tight transportation obstacles, to understand the potential causes of shortages and to formulate applicable strategies to deal with these problems. A literature review was then executed to identify previous shortages, with the primary focus being the most recent global shortage in 2009-2010. The literature review concentrated on the global supply of ${ }^{99} \mathrm{Mo}$, and information was gained from the perspectives of the Organisation for Economic Co-operation and Development/Nuclear Energy Agency, The Nuclear Science Advisory Committee, nuclear pharmacies, and hospitals $(3,5)$. All of the articles used contained current information and were published within the last $3 \mathrm{y}$.

Next, nuclear medicine technologists and nuclear pharmacists at the Mayo Clinic were interviewed. The purpose of these interviews was to obtain the approaches established by the nuclear medicine department at the Mayo Clinic to cope with the 2009-2010 shortage crisis and to evaluate the effectiveness of these approaches. If a shortage were to occur again, would they react similarly or would new strategies be implemented?

Lastly, survey questionnaires and phone interviews were conducted with 8 outside sources, including supervisors at outside nuclear medicine departments and nuclear pharmacists and managers at commercial nuclear pharmacies across the United States. Each outside source was asked to provide its Top 3 coping strategies for a ${ }^{99} \mathrm{Mo} /{ }^{99 \mathrm{~m}} \mathrm{Tc}$ shortage. All the coping strategies were then compiled and evaluated on the basis of effectiveness for procuring ${ }^{99 \mathrm{~m}} \mathrm{Tc}$ or conserving ${ }^{99 \mathrm{~m}} \mathrm{Tc}$ while still meeting the needs of the patients.

\section{RESULTS}

The viable responses were placed into 6 categories of coping strategies, including contractual agreements with commercial nuclear pharmacies, alternative imaging protocols, changes in imaging schedules, software use, generator management, and reduction of ordered doses or elimination
TABLE 1

Adoption Rates for the 6 Categories of Viable Coping Strategies

\begin{tabular}{lc}
\hline \multicolumn{1}{c}{ Strategy } & Adoption rate (\%) \\
\hline Changes in imaging schedules & 100 \\
Reduction of ordered doses or & 100 \\
elimination of backup doses & \\
Alternative imaging protocols & 75 \\
Generator management & 37.5 \\
$\begin{array}{l}\text { Contractual agreements with } \\
\text { commercial nuclear pharmacies }\end{array}$ & 25 \\
Software use & 12.5 \\
\hline
\end{tabular}

of backup doses (Table 1). These responses are listed on the basis of importance, with the most critical strategy first. They are also broad in terms for the nuclear medicine world and vary based on department size.

Table 1 shows the 6 categories in descending order according to adoption rate. The adoption rate refers to the percentage of the sources that indicated using that coping strategy during a shortage. Of the 8 sources from which strategies were obtained, $100 \%$ of them adopted both making changes in imaging schedule and reducing ordered doses or eliminating backup doses as a necessary step to cope with a shortage. Implementing alternative imaging protocols was a strategy that most $(75 \%)$ of the sources practiced during a ${ }^{99 \mathrm{~m}} \mathrm{Tc} /{ }^{99} \mathrm{Mo}$ shortage, and $37.5 \%$ of the sources mentioned generator management as being critical. A contractual agreement was disclosed by 2 sources as being the most important strategy for dealing with a shortage, which led to further investigation on the relationship between nuclear medicine departments and commercial nuclear pharmacies during a ${ }^{99} \mathrm{Mo} /{ }^{99 m} \mathrm{Tc}$ shortage. Finally, software use was found to be a Mayo Clinic-specific approach. Although the Mayo Clinic was the only source that acknowledged software use as being a key strategy, software use is necessary to optimize the use of ${ }^{99 \mathrm{~m}} \mathrm{Tc}$ when the resource is scarce.

Although each outside source was asked to provide its Top 3 coping strategies for a ${ }^{99} \mathrm{Mo} /{ }^{99} \mathrm{~m}$ Tc shortage, some sources provided more than 3 , suggesting that the strategies were all equally important. Therefore, these strategies were included in the adoption rates.

\section{DISCUSSION}

\section{Contractual Agreements with Commercial Nuclear Pharmacies}

When selecting a nuclear pharmacy for radiopharmaceutical services, cost difference, customer service, preferablecustomer status, and proprietary products are among items to be considered. However, before signing a contract with a commercial nuclear pharmacy, it is important to look at the ${ }^{99}$ Mo supply chain. In the United States, there are 3 generator manufacturers that are approved by the Food and Drug Administration: GE Healthcare (DryTec), Lantheus Medical Imaging (Technelite), and Mallinckrodt (Ultra-TechneKow DTE) (6). 
When preparing for a shortage, both nuclear medicine departments and nuclear pharmacies should know how and where the ${ }^{99 \mathrm{~m}} \mathrm{Tc}$ was obtained. DryTec is used only in GE Healthcare commercial nuclear pharmacies. Other commercial nuclear pharmacies use either a Technelite (Lantheus) or an Ultra-TechneKow DTE (Mallinckrodt) generator for ${ }^{99 \mathrm{~m}} \mathrm{Tc}$. Both of these generator manufacturers obtain their ${ }^{99} \mathrm{Mo}$ from multiple sources. Lantheus receives ${ }^{99} \mathrm{Mo}$ from 4 major processors and 6 of the 7 associated reactors, and Mallinckrodt receives its ${ }^{99}$ Mo from 5 major world suppliers $(2,7)$. This diversity allows stability in the ${ }^{99}$ Mo supply if a source is interrupted. Although there is flexibility in the supply chain, shortages can still occur. Negotiating a contract that would allow the nuclear medicine department to be informed of its ${ }^{99}$ Mo supply and alerted to any changes that arise would allow time to prepare for potential shortages. An optimal contract may also give an institution higher priority when ${ }^{99 \mathrm{~m}} \mathrm{Tc}$ is scarce. Therefore, when negotiating a contract, the logistics and timing have to be carefully evaluated.

\section{Alternative Imaging Protocols}

A common approach used during shortages is implementing different imaging protocols, such as reducing radioactivity administered or using alternate radiopharmaceuticals. The technologic developments in $\gamma$-camera hardware allow for the potential for nuclear medicine examinations to be performed using less administered radioactivity. This is beneficial to the patient, in regards to radiation exposure, while cutting costs and making more efficient use of the available ${ }^{99 \mathrm{~m}} \mathrm{Tc}$. Reducing doses is a common approach; however, it does have some drawbacks. Image quality is reduced as a result of lower counting rates; therefore, imaging time must be increased to accommodate for this. Increasing imaging time can then result in a chain of events, affecting camera availability, patient volumes, and scheduling.

Using alternate radiopharmaceuticals is often a better approach because it decreases the demand for ${ }^{99 \mathrm{~m}} \mathrm{Tc}$ while the same information is obtained from the images. One example would be using ${ }^{201} \mathrm{Tl}$-thallous chloride instead of ${ }^{99 \mathrm{~m} T c-s e s t a m i b i}$ or ${ }^{99 \mathrm{~m}} \mathrm{Tc}$-tetrofosmin for myocardial perfusion imaging. Thallium is a potassium analog that is constantly pumped in and out of the myocardial cells via the sodium-potassium pump, making it an ideal agent for myocardial perfusion imaging. Because of its redistribution properties, ${ }^{201} \mathrm{Tl}$ may also be used for detecting viable tissue in the heart, which helps determine whether the myocardium can be reperfused with surgery. Because of some disadvantagessuch as a longer half-life $(73.1 \mathrm{~h})$ relative to ${ }^{99 \mathrm{~m}} \mathrm{Tc}$, resulting in higher radiation exposure to patients; poorer image quality due to the low-energy mercury $\mathrm{x}$-rays in the 68 - to $80-\mathrm{keV}$ range emitted during the decay of ${ }^{201} \mathrm{Tl}$; and the need to have a camera available for immediate poststress imaging before redistribution occurs to avoid false-negative findings $-{ }^{201} \mathrm{Tl}$ is not a routinely used radiopharmaceutical. An advantage of ${ }^{201} \mathrm{Tl}$-thallous chloride is that it is relatively inexpensive compared with ${ }^{99 \mathrm{~m}} \mathrm{Tc}$; however, imaging with ${ }^{201} \mathrm{Tl}$ in some cases could result in an increased need for downstream testing, increasing both cost and patient radiation exposure (8). Another example would be using ${ }^{111}$ In-labeled leukocytes instead of ${ }^{99 \mathrm{~m}} \mathrm{Tc}$-labeled leukocytes for infection imaging, including sites of inflammatory bowel disease and osteomyelitis. Although ${ }^{99 \mathrm{~m}} \mathrm{Tc}$ 's photon energy, half-life, image quality, and lowered absorbed radiation dose to the patient are more ideal characteristics for a radiopharmaceutical, imaging with ${ }^{111}$ In-labeled leukocytes has its advantages. The biodistribution of ${ }^{111} \mathrm{In}$-labeled leukocytes is different from that of ${ }^{99 \mathrm{~m}} \mathrm{Tc}$-labeled leukocytes. Unlike with ${ }^{99 \mathrm{~m}} \mathrm{Tc}$-labeled leukocytes, early imaging of the abdomen and pelvis is not critical because ${ }^{111}$ In-labeled leukocytes do not normally accumulate in the bowel. ${ }^{111} \mathrm{In}$-labeled leukocytes are also advantageous in the case of osteomyelitis because simultaneous ${ }^{111}$ In-labeled leukocyte/99m Tc-medronate bone images may be obtained using multiple photopeak windows to differentiate the photons.

\section{Changes in Imaging Schedules}

Changes in imaging schedules include extending hours of operation; postponing, grouping, and rescheduling patients; and eliminating examinations. Extending hours of operation during periods of ${ }^{99 \mathrm{~m}} \mathrm{Tc}$ shortages beyond normal business hours or weekends makes more efficient use of the available ${ }^{99 \mathrm{~m}} \mathrm{Tc}$. For example, patient schedules may be arranged to correspond to generator deliveries and elutions, allowing additional patients to be imaged on hot days when more ${ }^{99 \mathrm{~m}} \mathrm{Tc}$ is obtainable. Although available ${ }^{99 \mathrm{~m}} \mathrm{Tc}$ can be more efficiently used by extending hours, complications can arise with employee morale and facility hours of operation policies. These should both be considered if proposing hours of operation changes for a nuclear medicine department.

Postponing, grouping, and rescheduling patients allows for both ${ }^{99 \mathrm{~m}} \mathrm{Tc}$ and kits to be saved by dedicating certain days of the week to certain examinations. An example of this would be performing bone scans only on Mondays, Wednesdays, and Fridays and performing renal function imaging ( ${ }^{99 \mathrm{~m}} \mathrm{Tc}-$ mertiatide or ${ }^{99 \mathrm{~m}} \mathrm{Tc}$-pentetate) on Tuesdays and Thursdays. Even though this decreases patient flexibility in scheduling, this would allow maximum use of the ${ }^{99 \mathrm{~m}} \mathrm{Tc}$-medronate (or ${ }^{99 \mathrm{~m}} \mathrm{Tc}$-oxidronate) kits by the nuclear pharmacy on Mondays, Wednesdays, and Fridays and eliminate the need for this kit to be compounded on other days of the week. When postponing, grouping, and rescheduling patients, the emergent nature of the examinations needs to be considered. Examinations such as myocardial perfusion imaging should not be grouped, but rather should be available to be performed daily because of its use in demonstrating myocardial ischemia and hibernating and stunned myocardium. Renal and bone imaging examinations are often less emergent and, therefore, may be grouped, limiting the amount of kits needing to be compounded daily and thus reducing the need for ${ }^{99 \mathrm{~m}} \mathrm{Tc}$. 
Eliminating examinations may also be a necessary step to reduce ${ }^{99 \mathrm{~m} T c}$ waste. An example of a study that results in a large amount of ${ }^{99} \mathrm{~m}$ Tc waste is periictal imaging of epilepsy patients. Nuclear medicine imaging has proven to be effective in locating seizure foci in the brain, using cerebral blood flow radiopharmaceuticals $\left({ }^{99 \mathrm{~m}} \mathrm{Tc}-\right.$-exametazime or ${ }^{99 m}$ Tc-bicisate) that have a rapid, first-pass extraction. This involves performing ictal and interictal SPECT imaging to identify a region of hyperperfusion in patients with focal epilepsy (9). For ictal imaging, a dose needs to be readily available for intravenous injection on the sudden onset of a seizure, so an image can be produced showing the cerebral perfusion pattern that was present soon after the injection of the radiopharmaceutical (9). Although this is an important clinical application, periictal imaging leads to unused doses, resulting in ${ }^{99 \mathrm{~m}} \mathrm{Tc}$ waste.

As an alternative to nuclear medicine, other noninvasive, diagnostic imaging modalities may be used, such as CT and MRI. These diagnostic procedures are used to detect anatomic changes, whereas nuclear medicine procedures often identify physiologic changes associated with disease. Often times, nuclear medicine can detect changes before any anatomic changes occur, allowing early identification of diseases and early evaluation of responses to therapeutic treatments (10). In the case of ictal/interictal imaging, CT, MRI, and lumbar puncture may be used to localize the site of seizure onset (11). Although there are alternative modalities for localizing the epileptogenic focus, nuclear medicine proves to be the most superior modality, and therefore these other modalities will not replace nuclear medicine ictal/interical imaging. In the case of cardiac imaging, there are 6 different procedures that can be used for diagnosing cardiac ischemia: myocardial perfusion imaging SPECT, cardiac CT angiography, stress echocardiography, cardiac PET myocardial perfusion imaging, coronary artery calcium scoring, and cardiac myo-

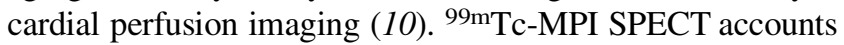
for $72 \%$ of cardiac imaging procedures in the United States, making it the most used cardiac imaging procedure (10). While determining which diagnostic modality to use for diagnosing cardiac ischemia, infrastructure (equipment and expertise), cost, and amount of reimbursement are more favorable factors for myocardial perfusion imaging SPECT (10).

There are some examinations in which nuclear medicine provides unique clinical information and no alternatives exist. These studies should not be eliminated, but rather prioritized. This includes lung ventilation and perfusion imaging in patients with suspected pulmonary emboli, lymphoscintigraphy for sentinel lymph node localization, and identification of parathyroid adenomas. Although these studies require higher priority, changes may still be made, such as reducing administered dose and grouping and rescheduling patients. For example, coordinating with surgical staff to schedule lymphoscintigraphy patients the same day as surgery, rather than next-day surgery, can reduce the ${ }^{99 \mathrm{~m}} \mathrm{Tc}$ in the dose.

\section{Software Use}

Specifically designed nuclear pharmacy software applications should be used to calculate theoretic activity and track ${ }^{99 \mathrm{~m}} \mathrm{Tc}$ usage by a department over time. Software programs that are commonly used to simplify ordering, streamline inventory receipts, and manage product documentation include $\mathrm{ec}^{2}$ Software Solutions applications, such as, BioRx (designed for nuclear pharmacies) and BioDose (designed for nuclear medicine departments), Syntrac Integration Tools by Cardinal Health, and Optility by Capintec Inc. (12-14). The initial cost, training required to learn a new system, and additional time necessary to document ${ }^{99 \mathrm{~m}} \mathrm{Tc}$ usage are minimal compared with anticipated benefits of using these applications.

Although these software applications solely monitor ${ }^{99 \mathrm{~m}} \mathrm{Tc}$ usage and provide no information about what should be done with the available ${ }^{99 \mathrm{~m}} \mathrm{Tc}$, they can be used to improve the selection of generator size and delivery schedules, if used applicably. Nuclear medicine technologists and nuclear pharmacists need to use the information gained from the software to make decisions about kit preparation and planning schedules. By using software applications to aid in decision making, the use of daily and weekly ${ }^{99 \mathrm{~m}} \mathrm{Tc}$ radioactivity can be optimized for more efficient use of the available resource while reducing costs.

\section{Generator Management}

Generator management is important in ${ }^{99} \mathrm{Mo} /{ }^{99} \mathrm{~m} \mathrm{Tc}$ shortages. Although it takes approximately $22.9 \mathrm{~h}$ for ${ }^{99 \mathrm{~m}} \mathrm{Tc}$ to reach maximum activity after elution, elutions may be performed before equilibrium is reached. Approximately $10 \%$ of the ${ }^{99 \mathrm{~m}} \mathrm{Tc}$ is produced per hour for the first $10 \mathrm{~h}$ after elution. Currently, many nuclear pharmacies elute their generators either once or twice a day. Increasing the frequency of generator elutions to several times throughout the day allows more activity to be obtained. As a result of more frequent elutions, more deliveries would be necessary.

While eluting a generator, fractionated elution is a technique that can be implemented to effectively obtain a high specific concentration of ${ }^{99 \mathrm{~m}} \mathrm{Tc}$ eluate. The volume of normal saline in the evacuated collecting vial can be altered based on the age of the generator to achieve a desired specific concentration. For example, when full elution yields are not needed, the volume of saline used for the elution can be decreased to increase the activity per volume concentration.

\section{Reduction of Ordered Doses or Elimination of Backup Doses}

Reducing the ordered doses or eliminating backup doses is a common first approach for shortage situations. During these times, nuclear medicine departments often choose to triage doses as needed, thus reducing or eliminating standing orders prepared by the nuclear pharmacy. Another way to reduce unnecessary ${ }^{99 \mathrm{~m}} \mathrm{Tc}$ usage is eliminating 
backup doses. Often times backup doses are ordered to be available for add-on patients. Although these doses provide the opportunity for nuclear medicine departments to image add-on patients, oftentimes they go unused and decay away. Therefore, in shortage situations nuclear medicine departments should eliminate add-on orders to conserve ${ }^{99 \mathrm{~m} T c}$.

\section{CONCLUSION}

The ${ }^{99} \mathrm{Mo} /{ }^{99 m} \mathrm{Tc}$ situation is continuously changing, so it is necessary to closely monitor it. A nuclear medicine department should establish standard operating procedures that can be promptly implemented if a shortage would occur. During a ${ }^{99} \mathrm{Mo} /{ }^{99} \mathrm{~m} \mathrm{Tc}$ shortage, it is important to adequately manage the situation. Therefore, it is essential that a strong collaboration be formed between nuclear medicine departments and nuclear pharmacies. If both nuclear medicine departments and nuclear pharmacies preemptively implement these viable strategies, ${ }^{99 \mathrm{~m}} \mathrm{Tc}$ usage can be maximized during shortage situations.

\section{DISCLOSURE}

No potential conflict of interest relevant to this article was reported.

\section{ACKNOWLEDGMENT}

This paper's abstract was presented at the annual meeting of the Society of Nuclear Medicine and Molecular Imaging in San Diego, California, on June 13, 2016.

\section{REFERENCES}

1. Radioisotopes in medicine. World Nuclear Association website. http://www. world-nuclear.org/information-library/non-power-nuclear-applications/radioisotopes-research/radioisotopes-in-medicine.aspx. Updated December 28, 2016. Accessed January 30, 2017.
2. Nuclear imaging: global Mo-99 supply chain. Mallinckrodt Pharmaceuticals website.http://www2.mallinckrodt.com/nuclear_imaging//global_mo-99_supply. aspx. Updated 2015. Accessed January 11, 2017.

3. Nuclear Energy Agency-Organisation for Economic Co-operation and Development. Medical isotope supply in the future: production capacity and demand forecast for the ${ }^{99} \mathrm{Mo} /{ }^{99 \mathrm{~m}} \mathrm{Tc}$ market, 2015-2020. NEA report NEA/SEN/HLGMR (2014) 2. Issy-lesMoulineaux, France: Nuclear Energy Agency; 2014.

4. Eczacıbaşı Monrol. Eczacıbaşı Monrol website. http://www.monrol.com.tr/en/29/ product-details/montek-mo99tc99m-generator. Accessed January 30, 2017.

5. Nuclear Science Advisory Committee. Annual assessment of the NNSA-material management and minimization $\left(\mathrm{M}^{3}\right) \mathrm{Mo}^{99}$ program. Nuclear Science Advisory website. http://science.energy.gov/ /media/np/nsac/pdf/docs/2015/Mo-99_2015_ Final_07-30-15.pdf. Published July 30, 2015. Accessed January 11, 2017.

6. Drugs@FDA: FDA approved drug products. Food and Drug Adminstration website. http://google2.fda.gov/search?as_sitesearch=www.accessdata.fda.gov/scripts/ cder/daf/index.cfm\&q=tc-99m+generator+companies\&client=FDAgov\&proxystylesheet=FDAgov\&output=xml_no_dtd\&site=FDAgov\&requiredfields=-archive: Yes\&sort=date:D:L:d1\&filter $=1 \&$ ie $=$ UTF-8\&ulang=en\&\&access $=$ p\&entq$\mathrm{r}=1 \&$ entqrm $=0 \& \mathrm{wc}=200 \& \mathrm{wc} \_\mathrm{mc}=1 \& \mathrm{oe}=\mathrm{UTF}-8 \& \mathrm{ud}=1$. Accessed January 30, 2017.

7. Lantheus' low-enriched uranium (LEU) TechneLite generator. TechneLite website. http://www.technelite.com/home.html. Accessed January 11, 2017.

8. Small GR, Ruddy TD, Simion O, et al. lessons from the Tc-99m shortage: implications of substituting Tl-201 for Tc-99m single-photon emission computed tomography. Circ Cardiovasc Imaging. 2013;6:683-691.

9. Cascino GD, So EL, Buchhalter JR, Mullan BP. The current place of single photon emission computed tomography in epilepsy evaluations. Neuroimaging Clin N Am. 2004;14:553-561.

10. Committee on State of Molybdenum-99 Production and Utilization and Progress Toward Eliminating Use of Highly Enriched Uranium, Nuclear and Radiation Studies Board, Division on Earth and Life Studies, National Academies of Sciences, Engineering, and Medicine. Molybdenum-99 for Medical Imaging. Washington, DC: The National Academies Press; 2016.

11. Epilepsy. Radiological Society of North America, Inc. website. http://www.radiologyinfo. org/en/info.cfm?pg = epilepsy. Published May 26, 2015. Accessed January 11, 2017.

12. ec2 Software Solutions: software for the nuclear medicine and molecular imaging fields. ec2 Software Solutions website. http://www.ec2software.com/. Accessed January 11, 2017.

13. Syntrac Integration Tools. Cardinal Health website. http://www.cardinalhealth. com/en/product-solutions/pharmaceutical-products/nuclear-medicine/businessand-practice-management/syntrac-integration-tools.html. Accessed January $11,2017$.

14. Optility Nuclear Medicine Management Software. Capintec Inc. website. http://www.capintec.com/product/optility/. Published 2016. Accessed January $11,2017$. 